Nachrichten

https://doi.org/10.1515/nf-2018-0005

\title{
Einladung zur Mitgliederversammlung während des FENS Forum 2018 in Berlin (7.-11. Juli 2018)
}

Termin: Sonntag, 8. Juli 2018, 18:45-21:30 Uhr

Ort: $\quad$ CityCube Berlin

\section{Vorläufige Tagesordnung:}

1. Begrüßung durch den Präsidenten

2. Bestätigung des Protokolls der letzten Mitgliederversammlung

3. Bericht des Schatzmeisters

4. Mitteilungen

5. Aktivitäten der Gesellschaft

6. Verschiedenes
Vorschläge für weitere Tagesordnungspunkte müssen bis spätestens 15. Juni 2018 bei der Geschäftsstelle eingegangen sein.

Neurowissenschaftliche Gesellschaft e. V. Max-Delbrück-Centrum für Molekulare Medizin (MDC) Robert-Rössle-Str. 10 13092 Berlin

E-Mail: gibson@mdc-berlin.de

\section{NWG-Reisestipendien für das FENS Forum 2018 in Berlin vergeben}

Aus den zahlreichen Bewerbungen aus In- und Ausland wurden nun die Gewinner eines Reisestipendiums für die Teilnahme am FENS Forum 2016 in Berlin ausgewählt.

- Thordis Arnold (Kassel)

- Antonia Beiersdorfer (Hamburg)

- David Bergin (Hannover)

- Dominik Dannehl (Mannheim)

- Nina Hahn (Göttingen)

- Fatemeh Hassani Nia (Hamburg)

- Stefanie Hauck (Bonn)

- Sarah Joost (Rostock)

- Merryn Jordan (Mannheim)

- Shokoufeh Khakipoor (Freiburg)

- Antje Kilias (Freiburg)

- Stanislava Knyazeva (Magdeburg)

- Ina Leiter (Hannover)

- Constanze Lenschow (Lisabon)

- Thomas Lissek (Heidelberg)
- Anne Stephanie Mallien (Mannheim)

- Sabina Marciano (Tübingen)

- Daniel Nicolae Mitroi (Madrid)

- Moritz Oberstadt (Leipzig)

- Michael Rabenstein (Rostock)

- Phillip Rieder (Homburg)

- Nicole Rosskothen-Kuhl (Freiburg)

- Annabelle Schlüter (Heidelberg)

- Akash Srivastava (Jena)

- Bharath Chandra Talluri (Hamburg)

- Winnie Wefelmeyer (London)

- Lisa Welzel (Hannover)

Herzlichen Glückwunsch!

Das Stipendium in Höhe von 500 Euro wird in bar am NWG-Stand auf dem FENS Forum in Berlin gegen Vorlage eines Ausweises ausgezahlt. 


\section{Vorstandswahl für die Amtsperiode 2019-2021}

Laut Satzung ist im Januar 2019 die Wahl des NWG-Vorstandes für die Amtsperiode 2019-2021, die mit dem Ende der Göttinger Tagung am 23. März 2019 beginnen wird, fällig.

Alle Mitglieder sind aufgefordert, Vorschläge für die Positionen der Sektionssprecher, des Schatzmeisters, des Generalsekretärs und des Vizepräsidenten bei der Geschäftsstelle einzureichen.

Das Amt des Präsidenten steht nicht zur Wahl, laut Satzung wird der Vizepräsident der vorangegangenen Amtsperiode automatisch Präsident der nächsten Amtsperiode.
Es können nur Vorschläge berücksichtigt werden, die die komplette postalische Adresse, die Telefonnummer und die E-Mail-Adresse des Kandidaten enthalten.

Der Stichtag für die Einsendung von Vorschlägen ist der 3. September 2018.

Vorschläge per E-Mail an:

Meino Alexandra Gibson

E-Mail: gibson@mdc-berlin.de

Die Vorschläge werden von der Wahlkommission der NWG für die endgültige Wahlliste gesichtet und bei Bedarf ergänzt werden.

\section{Fortbildungsprogramme der Neurowissenschaftlichen Gesellschaft 2018/2019 - Von Mitgliedern für Mitglieder}

Es ist wieder Zeit, Vorschläge für die Methodenkurse und auch die Lehrerfortbildungen der NWG zu sammeln. Diese sind seit Langem eine feste Einrichtung und erfreuen sich großer Beliebtheit. Die Mitglieder der NWG sind aufgefordert, derartige Kurse, für die die NWG eine finanzielle Unterstützung bereitstellt, im kommenden Jahr anzubieten.

Für die Methodenkurse stellt die NWG $125 €$ pro teilnehmendem NWG-Mitglied und 62,50€ pro teilnehmendem Nicht-Mitglied bis zu einer maximalen Höhe von $2.500 €$ pro Kurs zur Verfügung. Die Lehrerfortbildungsveranstaltungen werden mit einem Betrag in Höhe von maximal $250 €$ pro Veranstaltung unterstützt.

Beide Programme werden mit einem gedruckten Plakat bzw. gedruckten Flyern im Spätsommer des Vorjahres angekündigt. Das Lehrerfortbildungsprogramm erstreckt sich über ein Schuljahr, also von September 2018 bis Juli 2019, das Methodenkursprogramm über das Kalenderjahr 2019.

Einsendeschluss für Angebote ist Montag, der 2. Juli 2018.

Details können bei der Geschäftsstelle der NWG erfragt werden (gibson@mdc-berlin.de).

\section{Weitere Informationen:}

Methodenkurse 2019: http://nwg-info.de/aktivitaeten/ kurse_workshops/2018 Lehrerfortbildungen 2017/2018: http://nwg-info.de/de/ aktivitaeten/lehrerfortbildung/2018 


\section{Neueintritte}

Folgende Kolleginnen und Kollegen dürfen wir als Mitglieder der Neurowissenschaftlichen Gesellschaft begrüßen:

Abd El Hay, Muad (Heidelberg)

Ackermann, Dr. Frauke (Berlin)

Adolf, Andrej (Berlin)

Agarwal, Dr. Amit (Heidelberg)

Ahmed, Amina (Stuttgart-Leinfelden)

Aksoy-Aksel, Dr. Ayla (Tübingen)

Alatriste González, Diana Karina (Berlin)

Araragi, Dr. Naozumi (Berlin)

Araujo Sousa, Carolina (Heidelberg)

Bach, Annika (Aachen)

Barker, Dr. Alison (Berlin)

Barnstedt, Dr. Oliver (Bonn)

Bégay-Müller, Dr. Valérie (Berlin)

Bell, Christopher (Mainz)

Bengoetxea Bausela, PhD Xabier (Münster)

Bergeler, Dr. Jürgen (Berlin)

Berghoff, Stefan (Göttingen)

Bergin, Dr. David (Hannover)

Biel, Anna Lena (München)

Braun, Anke (Hamburg)

Brembs, Prof. Dr. Björn (Regensburg)

Brickwedde, Marion (Bochum)

Brosch, Marcel (Magdeburg)

Bucher, Michael (Hamburg)

Camblor Perujo, Santiago Manuel (Essen)

Chang, Hae In (Tübingen)

Chen, Changwan (Köln)

Cortes Campo, Orlando Jose (Würzburg)

Dannehl, Dominik (Mannheim)

De la Flor Aguirre, Magin (Berlin)

Delettre, Céline (Hamburg)

Demiray, Yunus Emre (Magdeburg)

D’Errico, Dr. Anna (Frankfurt)

Detering, Nora Tula (Hannover)

Di Benedetto, Dr. Barbara (Regensburg)

Dietzmeyer, Nina (Hannover)

Dolón Vera, Laura (Bochum)

Dunay, Prof. Dr. Ildiko Rita (Magdeburg)

Eckl, Nina (Mainz)

Egger, Prof. Dr. Veronica (Regensburg)

El Sayed, Prof. Nesrine (Cairo, Egypt)

Engelhardt, Dr. Maren (Mannheim)

Ernst, Laura (Bochum)

Evangelia, Pollali (Magdeburg)

Fatima, Nida-Ul (Berlin)

Fecko, Dr. Miroslav (Bochum)
Felix, Lisa (Düsseldorf)

Flüh, Dr. Charlotte (Kiel)

Fraize, Dr. Nicolas (Bochum)

Franco Emch, Monica (München)

Garza Gomez, Alejandra Patricia (Magdeburg)

Grömmke, Michelle (Bochum)

Haak, Annika (Bochum)

Hacker, Daniela (Hamburg)

Hahn, Nina (Göttingen)

Hauck, Stefanie (Bonn)

Haumann, Dr. Iris (Hamburg)

Haupt, Marleen (München)

Hauser, Maximilian (Bochum)

Heinrich, Laurin (Mainz)

Hogekamp, Zarina (Magdeburg)

Hurtado Zavala, Dr. Joaquin Isaac (Hamburg)

Ikhsan, Maulana (Lübeck)

Jack, Alexander (Bochum)

Johanns, Ole (Hamburg)

Jordan, Merryn (Mannheim)

Kilo, Lukas (Mainz)

Klausch, Monique (Magdeburg)

Kleidonas, Dimitrios (Freiburg)

Klein, Alexandra (Martinsried)

Knyazeva, Stanislava (Magdeburg)

Koduah, Priscilla (Berlin)

Kollert, Dr. Sina (Würzburg)

Konietzny, Anja (Hamburg)

Kowalczyk, Izabela (Berlin)

Kronenberg-Versteeg, Dr. Deborah (Cambridge, UK)

Krueger-Burg, Dr. Dilja (Göttingen)

Krüger, Marie (Bochum)

Kubinski, Sabrina (Hannover)

Kücükerden, Melike (Hannover)

Kühnemund, Johannes (Berlin)

Kul, Ufuk Emre (Magdeburg)

Laier, Alena (Berlin)

Lang, Daniel (Magdeburg)

Langenhagen, Alina Katharina (Hannover)

Laprell, Dr. Laura (Hamburg)

Larkum, Prof. Dr. Matthew (Berlin)

Leiter, Dr. Ina (Hannover)

Lischka, Katharina (Freising)

Lissek, Thomas (Heidelberg)

Litke, Christian (Heidelberg)

Lozano Navarro, Dr. Yavé Roberto (Würzburg)

Lupp, Julia (Frankfurt/Main)

Malci, Ayse (Magdeburg)

Marciano, Sabina (Tübingen) 
Martin, Christian-David (Kensington, UK)

Martinez, Erick (Bonn)

Masch, Jennifer-Magdalena (Göttingen)

Mazaraki, Dimitra (Berlin)

Mecklenburg, Dr. Nora (Berlin)

Méndez-Couz, Dr. Marta (Bochum)

Meschkat, Martin (Göttingen)

Morton Moreno, Lorena (Magdeburg)

Müller, Dr. Anke (Magdeburg)

Müller, Dr. Miriam (Frankfurt / Main)

Mylius, Dr. Judith (Göttingen)

Nieter, Felix (Göttingen)

Oberländer, Victoria (Hamburg)

Oberstadt, Dr. Moritz (Leipzig)

Oberwinkler, Prof. Dr. Johannes (Marburg)

Özcan, Ismarl (Berlin)

Paetz, Christina (Leipzig)

Patrizi, PhD Annarita (Heidelberg)

Petese, Alessandro (Bonn)

Peylo, Charline (München)

Pfeffer, PhD Thomas (Hamburg)

Quiceno, Gloria (Magdeburg)

Radecke, Jan-Ole (Hamburg)

Raza, Syed Ahsan (Magdeburg)

Reid, Dr. Anna (Berlin)

Renz, Pia Malika (Berlin)

Rifat, Ali (Berlin)

Ripp, Isabelle (München)

Rodriguez-Rozada, Silvia (Hamburg)

Roll, Dr. Lars (Bochum)

Rumpf, Florian (Würzburg)

Samehni, Mohamad (Köln)

Sammons, Dr. Rosanna (Berlin)

Sánchez Carranza, Oscar (Berlin)

Scharrenberg, Robin (Hamburg)

Schlueter, Dr. Annabelle (Heidelberg)

Schmartz, Mylène (Berlin)

Schnepel, Dr. Philipp (Berlin)

Schölvinck, Dr. Marieke (Frankfurt / Main)

Schwaller, Dr. Fred (Berlin)

Schweizer, Dr. Renate (Göttingen)

Schwitzer, Susanne (Berlin)
Sethumadhavan, Nithya (Bochum)

Signoret-Genest, Dr. Jérémy (Würzburg)

Silveira Broggini, PhD Ana Clara (Frankfurt / Main)

Sirmpilatze, Nikoloz (Göttingen)

Soba, Dr. Peter (Hamburg)

Sommer, Daniela (Diepenbeek, Belgium)

Song, Lingzhen (Hamburg)

Srivastava, Akash (Jena)

Stahlberg, Dr. Markus (Göttingen)

Stauch, Benjamin (Frankfurt / Main)

Steinhardt, Julia (Lübeck)

Stewart, Ian Erik (Berlin)

Subramanian, Swati (Göttingen)

Sushma, Dagar (Düsseldorf)

Süß, Sandra (Bochum)

Tayaranian Marvian, Amir (München)

Thiem, Jessica (Mainz)

Tong, Yixin (Freiburg i. Br.)

Tursun, Dr. Baris (Berlin)

Udhayachandran, Dr. Annapoorani (Berlin)

Uran, Cem (Frankfurt am Main)

van den Brink, PhD Ruud (Hamburg)

van Kronenberg, Philipp (Göttingen)

Vasilevska, Veronika (Magdeburg)

Voigt, Stefan (Berlin)

von Hövel, Friederike (Hannover)

Walter, Lisa Marie (Hannover)

Wefelmeyer, Dr. Winnie (London, UK)

Whitmire, Dr. Clarissa (Berlin)

Wilhelm, Nicole (Mainz)

Xia, Zifeng (Magdeburg)

$\mathrm{Xu}$, Dr. Xiaxia (Hamburg)

Yakoubi, Rachida (Jülich)

Yang, Wei (Hamburg)

Yildirim, Jun. Prof. Ferah (Berlin)

Zheng, Dr. Fang (Erlangen)

Zhong, Wei (Berlin)

Zuza, Kristina (Heidelberg)

Der Mitgliedsstand zum 19. März 2018 beträgt 2.268 Mitglieder. 


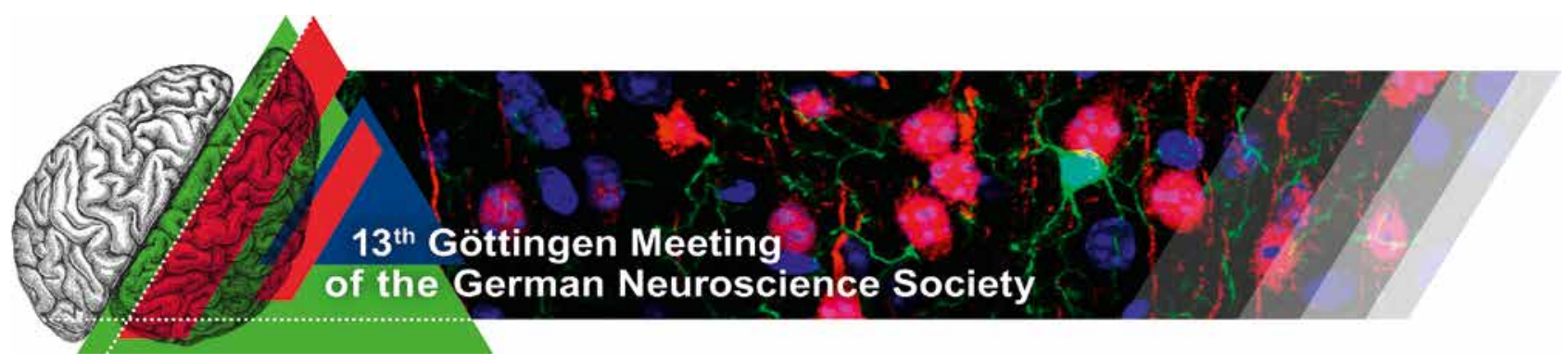

\section{0.-23. März 2019}

Das Programmkomitee für die Göttinger Tagung 2019 hat sich am 2. März 2018 in Berlin getroffen und aus den 46 eingereichten Vorschlägen 34 ausgewählt. Die Göttinger Tagung bietet insgesamt für 36 Symposien Raum. Zwei Plätze werden freigehalten für die „Breaking News“ Sessions, für die die Vorträge aus den im Herbst eingereichten Poster-Abstracts ausgewählt werden.

Adaptivity and inhomogeneity in neuronal networks - two sides of the same coin? Egert, Ulrich (Freiburg); Rotter, Stefan (Freiburg)

Behavioral decisions based on multimodal information

el Jundi, Basil (Würzburg); Strube-Bloss, Martin (Würzburg)

Beyond expression of fear: mechanisms and circuits of the extended amygdala

Lange, Maren (Münster); Seidenbecher, Thomas (Münster)

Brain-machine-interface in paralysis

Birbaumer, Niels (Tübingen)

Common principles of spatial and temporal sensory processing

Clemens, Jan (Göttingen); Martelli, Carlotta (Konstanz);

Silies, Marion (Göttingen)

Dissection of a central brain circuit: structure, plasticity and functions of the drosophila mushroom body Fiala, André (Göttingen); Gerber, Bertram (Magdeburg)

Early information selection for robust vision

Bethge, Matthias (Tübingen)
Form follows function? Rules and consequences of structural synaptic plasticity

Rose, Tobias (Martinsried); Wiegert, J. Simon (Hamburg)

From astrocytes to behaviors: searching the cellular and molecular roots of emotion dysfunctions

Di Benedetto, Barbara (Regensburg); Neumann, Inga (Regensburg)

From clinical symptoms to motoneuron pathobiology: most recent insights into amyotrophic lateral sclerosis (ALS)

Weishaupt, Jochen (Ulm); Ludolph, Albert C. (Ulm)

From normal brain development to pathology: what role does the environment play?

Cruceanu, Cristiana (München); Mayer, Simone (San

Francisco, USA)

Go with the flow? Processing of sensory flows across modalities

Arrenberg, Aristides (Tübingen); Benda, Jan (Tübingen); Denzinger, Annette (Tübingen); Mallot, Hanspeter (Tübingen)

Hearing system adaptation for diverse lifestyles across the animal kingdom

Nowotny, Manuela (Frankfurt/Main); Schöneich, Stefan (Leipzig)

Keeping neurons alive - erythropoietin, its variants and its receptors

Hahn, Nina (Göttingen); Heinrich, Ralf (Göttingen)

Mitochondrial dysfunction in neurodegeneration Milosevic, Ira (Göttingen); Raimundo, Nuno (Göttingen) 
Modulatory circuits of central pain processing Grinevich, Valery (Heidelberg); Groh, Alexander (Heidelberg)

Neural mechanisms of social decision-making Kagan, Igor (Göttingen); Pooresmaeili, Arezoo (Göttingen)

Neurodegenerative diseases: shaping neuronal circuits by membrane trafficking

Kononenko, Natalia (Köln); Wirth, Brunhilde (Köln)

Neurological autoimmunity: the role of pathogenic autoantibodies against neuron and glia proteins Moritz, Christian (Saint-Priest-en-Jarez, France); Sommer, Claudia (Würzburg)

Novel insights into the regulation of hypothalamic neurocircuits and functions

Fenselau, Henning (Köln)

Optogenetics - tool development and application in neuroscience

Gottschalk, Alexander (Frankfurt/Main)

Orexin beyond sleep

Fendt, Markus (Magdeburg); Koch, Michael (Bremen)

Inhibitory synapse diversity in health and disease Krueger-Burg, Dilja (Göttingen); Papadopoulos, Theofilos (Göttingen)

Pro-survival versus toxic NMDA receptor signaling and the fight against neurodegenerative disorders Bading, Hilmar (Heidelberg)

Resolving the cognitive function of prefrontal circuits: from neurons to behavior

Diester, Ilka (Freiburg); Hanganu-Opatz, Ileana (Hamburg)
Serotonin and its developmental role in shaping brain plasticity and neuropsychological phenotypes Alenina, Natalia (Berlin); Calabrese, Francesca (Milan, Italy); Popik, Piotr (Krakow, Poland)

Short-term adaptation in early auditory processing: from synaptic depression to focal perception Lingner, Andrea (Martinsried); Steculorum, Sophie (Köln)

Subcortico-cortical loops and their role in sensory processing and perception de Hoz, Livia (Göttingen); Hechavarria, Julio (Frankfurt/ Main)

The 4Rs in animal-based neuroscience research: Refinement, Reduction, Replacement, Responsibility Stilling, Roman (Münster); Treue, Stefan (Göttingen)

The brain oxytocin system - its complex impact on autism, social behavior, and stress

Jurek, Ben (Regensburg)

The dentate gyrus - from microcircuit function to control of behavior

Bartos, Marlene (Freiburg)

The neuronal basis of tinnitus

Mazurek, Birgit (Berlin); Schulze, Holger (Erlangen)

The presynaptic active zone: converging and diverging mechanisms across species

Kittel, Robert (Würzburg); Liphtein, Noa (Göttingen)

The tripartite synapse in health and disease Petzold, Gabor (Bonn); Rose, Christine (Düsseldorf)

Einreichungsschluss für Poster Abstracts ist der 1. Oktober 2018. 


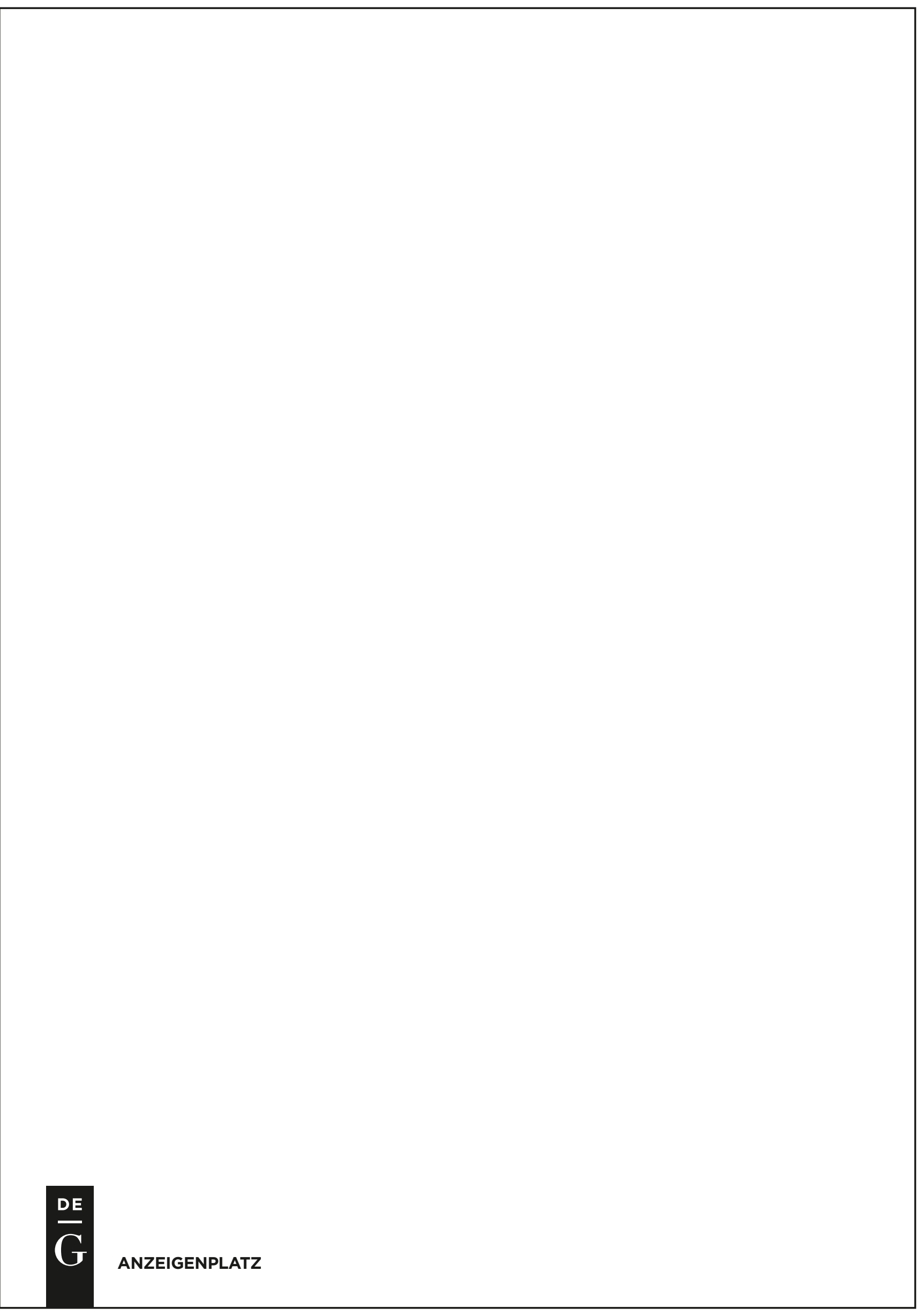




\section{FENS Forum 2018 in Berlin (7.-11. Juli 2018)}

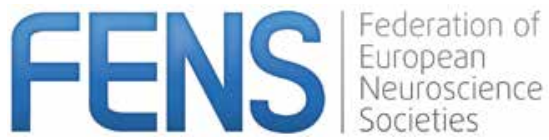

Der Einreichungsschluss für Poster-Abstracts für das FENS Forum in Berlin ist vorüber und wir wollen dies zum Anlass für einen kurzen Zwischenbericht nehmen. Die Anmeldezahlen sind überaus erfreulich: 5.700 Registrierungen und 4.700 Poster. Das sind jeweils etwa 1.000 mehr als für das FENS Forum 2016 in Kopenhagen, und damit erwarten wir eine Rekordbeteiligung. 20 Jahre nachdem das erste FENS Forum 1998 in Berlin stattfand, knüpfen wir an den Erfolg dieser Veranstaltung an.

\section{Introductory Courses}

Als ein Novum werden auf einem FENS Forum acht sogenannte „Introductory Courses“ angeboten, durchgeführt von Berliner PIs. Über 600 Teilnehmer (Stand: März 2018) haben sich angemeldet. Diese Workshops nach dem Motto „Everything you always wanted to know about ..." sollen junge Studenten oder auch Wissenschaftler, die sich in ein bisher unbekanntes Arbeitsgebiet einarbeiten möchten, eine breite und gut verständliche Einführung in eine Thematik geben. Sie finden am Freitag, den 6. Juli 2018, also vor Beginn des Forums, an verschiedenen Berliner Instituten statt. Die Themen sind „Alzheimer“, „Compu-
Das FENS Forum wartet nicht nur mit einem hervorragenden wissenschaftlichen Programm auf mit 24 Hauptvorträgen und 56 Symposien, sondern bietet auch zahlreiche Workshops, Special Interest, Satelliten- und Outreach Events an. $\mathrm{Zu}$ diesen leistet die NWG als gastgebende nationale Gesellschaft einen aktiven Beitrag. Stellvertretend organisiert das Host Society Committee (HSC) lokale Programme und Angebote für die Tagungsteilnehmer, um damit das FENS Forum noch attraktiver zu machen.

\section{Hertie-Stiftung -}

tational Neuroscience“, „Glial Cells“, „Invertebrates“, „Pain“, „Psychiatric Diseases“, „Retina“ und „Synaptic Transmission“. Derzeit gibt es noch einige freie Plätze bei „Glial Cells“ (Platzangebot wurde erweitert), „Pain“, „Invertebrates“ und „Retina“, so dass eine Anmeldung noch möglich ist, bis das Teilnehmerlimit erreicht ist. Die Gemeinnützige Hertie-Stiftung unterstützt dieses Programm.

https://forum2018.fens.org/forum-programme/introductory-courses\#.Wt721TMuCUk

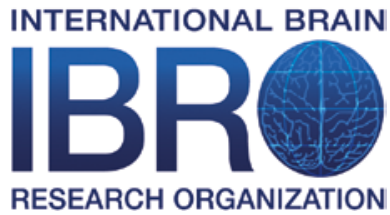

\section{Young Investigator Training Program (YITP) Lab Visits}

toranden finden. 27 deutsche Labore nehmen an diesem Programm teil und stellen ca. 45 Plätze bereit, für die sich knapp 200 Studenten beworben haben. IBRO gibt dafür Stipendien.

http://forum2018.fens.org/young-investigators/yitp-programme\#.WqjOLbMiHX4

\section{Blick hinter die Kulissen des Naturkundemuseum}

Das Naturkundemuseum bietet einer begrenzten Teilnehmerzahl Führungen in die Sammlungen des Museums an.
Die Anmeldung erfolgt über http://fensforum2018.nwginfo.de/registration.php 


\section{Special Interest Event „Alternative Careers for Neuroscientists“"}

Zusammen mit dem FENS Committee on Higher Education and Training und dem FENS Kavli Network of Excellence (FKNE) stellt die NWG in diesem Workshop berufliche Entwicklungsmöglichkeiten außerhalb der Wissenschaft vor. Neurowissenschaftler, die nach der akademischen Laufbahn einen anderen Weg eingeschlagen haben, berichten von ihrem Werdegang in der Industrie, im Verlag, im Wissenschaftsmanagement oder in der Privatwirtschaft.

http://forum2018.fens.org/forum-programme/special-interest-events\#.Wqj5rbMiHX4

\section{„History of Neuroscience“ - Abendverstaltung in der Virchow-Ruine}

In der Hörsaalruine des ehemaligen Rudolf-Virchow-Hörsaales im Berliner Medizinhistorische Museum findet am Sonntag, den 8. Juli von 19:00-22:30 Uhr ein Seminar zu "Germany's contribution to neuroscience in the $19^{\text {th }}$ and $20^{\text {th }}$ centuries" statt. Nach den Vorträgen werden Führungen durch die Virchow'sche Sammlung und die Aus-

\section{Jump the FENS}

Die traditionelle (Studenten-)Party des FENS Forums findet am Montag, den 9. Juli 2018 im legendären Soda-Club in der Kulturbrauerei am Prenzlauer Berg statt. Diese Party-Location bietet auf sechs Dance Floors unterschiedliche Musikrichtungen an und hat einen gro0en Außenbereich. Der Ticketpreis enthält nicht nur den Eintritt, sondern

\section{Opening Ceremony}

Langeweile soll bei der Eröffnungsveranstaltung nicht aufkommen. Lassen Sie sich überraschen!

Die Online Registrierung ist noch bis 20. Juni 2018 möglich, danach kann man sich auch noch direkt auf der Tagung registrieren. stellung des Medizinhistorischen Museums angeboten, abgerundet von einem Get-Together. Die Anmeldungen erfolgt auf http://forum2018.fens.org/forum-programme/ networking-events\#.Wqj4r7MiHX5. Die Teilnehmerzahl ist limitiert.

auch Getränke- und Essensbons. 1.000 Personen haben sich bereits angemeldet, das Soda bietet aber Raum für mehr als doppelt so viele Gäste.

https://forum2018.fens.org/young-investigators/jumpthe-fens\#.Wt73VjMuCUk

\section{Kontakt:}

Prof. Dr. Helmut Kettenmann

Chair Host Society Committee

Zelluläre Neurowissenschaften

Max Delbrück Center for Molecular Medicine (MDC) Berlin-Buch

Robert-Rössle-Str. 10

13125 Berlin

E-Mail: gibson@mdc-berlin.de

\section{FENS Fourm 2018 Host Society Committee:}

Helmut Kettenmann (Chair), Matthias Endres, Ulrich Dirnagl, Volker Haucke, Christine Heim, Andreas Heinz, Frank Heppner, Hans-Joachim Pflüger, Christian Rosenmund, Constance Scharff, Dietmar Schmitz,Stephan Sigrist, Verena Haage (Student), Constanze Holman (Student) 


\section{Ausstellung „SinnReich“}

Einen Beitrag zu Public Understanding of Science leistet die Ausstellung „SinnReich“, die vom 15. Juni bis 11. Juli 2018 im OttoBock Science Center am Potsdamer Platz stattfinden wird. Dieser Programmpunkt steht jedermann offen. Ziel der Ausstellung ist es, den Besuchern auf spannende, unterhaltsame und lehrreiche Weise die Funktionsweise der fünf Sinne des Menschen nahe bringen. Die interaktiven Exponate rund um das Thema Wahrnehmung
SCHERING

\section{\$ITIFTUNG Ottobock.}

werden vom Science Center „Turm der Sinne“ ausgeliehen. Die Ausstellung richtet sich an interessierte Laien, vor allem aber an Lehrer und ihre Schulklassen. Eine Lehrerfortbildung zum Thema rundet das Programm ab. Die Ausstellung kann mit der Unterstützung der ScheringStiftung verwirklicht werden.

http://hsc.nwg-info.de/sinnreich
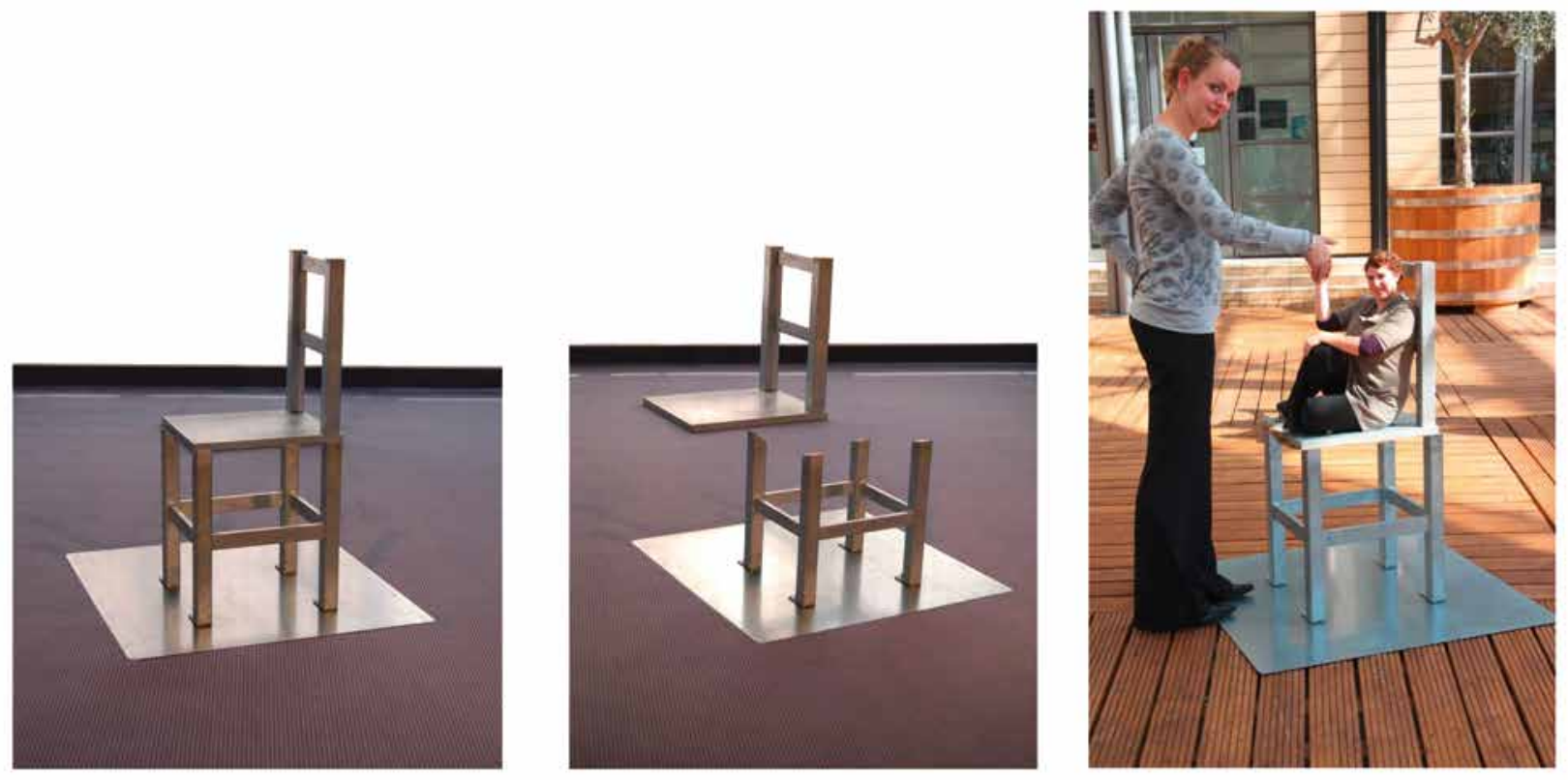

Abb.1: Beuchet-Stuhl. Exponat der Ausstellung „SinnReich“. Ausgeschaltete Größenkonstanz erzeugt Zwerg und Riese: durch ein Guckloch betrachtet, verschmelzen die beiden Teile zu einem gewöhnlichen Stuhl. Auf dem Stuhl scheint ein Zwerg zu sitzen und vor ihm steht ein Riese. Beim einäugigen Betrachten sind keine entscheidenden Tiefenmerkmale erkennbar. Deshalb deutet unser Gehirn die beiden Teile des Stuhls der Gewohnheit entsprechend als zusammengehörig, also als einen Stuhl. Die beiden Personen erscheinen folglich nebeneinander, aber unterschiedlich groß. Die biologisch sinnvolle Größenkonstanzleistung wird hier getäuscht: Normalerweise scheinen Gegenstände durch größere Entfernung nicht zu schrumpfen, obwohl ihr Bild auf der Netzhaut kleiner ist. Hier jedoch entsteht durch Mangel an räumlicher Tiefeninformation ein Riese und daneben ein Zwerg auf einem ganz normalen Stuhl.

Bildquelle: turmdersinne 


\section{Ausblick}

Für die nächsten Ausgaben von Neuroforum werden folgende Beiträge vorbereitet:

Special Issue: Hippokampale Langzeitpotenzierung (LTP) - Geschichte, Gegenwart und Zukunft

Klaus G. Reymann, Tim V. P. Bliss, Graham L. Collingridge und Richard G. M. Morris

Langzeitpotenzierung im Hippokampus:

Entdeckung, Mechanismen und Funktion

Denise Manahan-Vaughan

Die Regulation der hippocampalen Informationsenkodierung durch metabotrope Glutamatrezeptoren
Marina Mikhaylova und Michael R. Kreutz Geclusterte Plastizität bei LTP: Wie starke Synapsen bestehen bleiben, um Langzeitgedächtnis aufrecht zu erhalten

Detlef Balschun und Michael J. Rowan

Hippokampale Synaptische Plastizität bei Erkrankungen: $A ß$, tau und darüber hinaus

Volkmar Lessmann und Elke Edelmann Die Analyse synaptischer Plastizität auf Einzelzellebene mit Hilfe der STDP

\section{Preview}

The following contributions are in preparation for the next issues of Neuroforum:

Klaus G. Reymann, Tim V. P. Bliss, Graham L. Collingridge and Richard G. M. Morris

Long-term potentiation in the hippocampus:

discovery, mechanisms and function

Denise Manahan-Vaughan

Regulation of hippocampal information encoding by metabotopic glutamate receptors
Marina Mikhaylova and Michael R. Kreutz

Clustered plasticity in LTP: How strong synapses persist to maintain long-term memory

Detlef Balschun and Michael J. Rowan

Hippocampal synaptic plasticity in disease: rogue $A ß$, tau and beyond

Volkmar Lessmann and Elke Edelmann Analyzing synaptic plasticity at the single cell level with STDP 
